International Journal of Artificial Intelligence \& Applications (IJAIA), Vol.2, No.4, October 2011

\title{
APPLICATION OF IMPERIALIST COMPETITIVE ALGORITHM TO THE EMERGENCY MEDICAL SERVICES LOCATION PROBLEM
}

\author{
Saeed Moadi ${ }^{1}$, Afshin Shariat Mohaymany ${ }^{2}$ and Mohsen Babaei ${ }^{3}$ \\ ${ }^{1}$ Dept. of Civil Engineering, Iran University of Science and Technology, Tehran, Iran \\ saeedmoadi@gmail.com \\ ${ }^{2}$ Dept. of Civil Engineering, Iran University of Science and Technology, Tehran, Iran \\ shariateiust.ac.ir \\ 3 Dept. of Civil Engineering, Iran University of Science and Technology, Tehran, Iran \\ babaei@iust.ac.ir
}

\begin{abstract}
Minimization of arrival time at scenes plays an essential role to help injured people in emergency events. This can be undertaken through mathematical programming models, called emergency medical services location problem, and solved by conventional exact algorithms or by recent meta-heuristic methods as well. Meta-heuristic algorithms have recently been realized to be more efficient in the sense of computing times especially in large-scale cases. The emergency medical services location problem would be further complicated when the number of stations and/or emergency vehicles, as an important indicator of system costs, should be determined at the same time. In this paper, a newly introduced optimization method, Imperialist Competitive Algorithm (ICA), is used to solve the EMS location problem. The ICA mimics the human's socio-political evolution to solve continuous problems. In this paper, a discrete version of the ICA is sought to be adapted to solve the EMS location problem. The adapted ICA algorithm is then applied on two benchmark problems with four different demand scenarios as well as on the real transportation network of Mashhad City. Results of this algorithm are compared with those of other wellknow meta-heuristic algorithms (i.e. the genetic algorithm, the simulated annealing and the particle swarm optimization). These results indicate that the cpu time of the ICA is averagely less than that obtained from the other algorithms, and the number of required ambulances is not considerably different.
\end{abstract}

\section{KEYWORDS}

Imperialist Competitive Algorithm; Emergency Medical Services (EMS) location

\section{INTRODUCTION}

Almost all the problems concerning optimization issues in complex systems have a combinatorial nature. The vehicle routing problem, the warehouse or other facility location problems, and the transportation (or communication) network design problem are examples of complex and combinatorial optimization problems. In practice, the size of these problems is usually so large that one cannot obtain the global optimum solution for them with a polynomial run-time. However, these problems must be addressed even if the adopted algorithm turns over a suboptimal (local optima) solution. It should be noted that these sub-optimal solutions should be acceptable in terms of both time and quality. The adequacy of algorithm's performance is usually realized through experimenting on small size cases.

In different countries, to reduce morbidity and mortality caused by diseases and accidents, successful and efficient systems, usually called Emergency Medical Services (EMS) are designed. The task of such a system is to offer treatment services on event scenes and, if

DOI : 10.5121/ijaia.2011.2411 
International Journal of Artificial Intelligence \& Applications (IJAIA), Vol.2, No.4, October 2011

necessary, to move them from the scenes to medical centers. Due to increasing population growth, lack of facilities, limited capacity and system adequacy, strategies needed to be thought in this area even with the resources available to prevent emergency patients' mortality. Locating the emergency medical services is essential to reduce the response time. Thus, any reduction in the response time of emergency services will play an essential role in saving lives. Location models used in order to locate Emergency Medical Services' sites specify the number of ambulances needed at each location. In these models, the demand nodes usually obtained by aggregating emergency demands of neighbour regions (e.g., depending on the number of calls per day). In areas where it is possible to establish (open) a station (base) for a limited number of emergency medical services (vehicles) the use of potential nodes is suggested, among which the applied model seeks to deploy the least number of stations (ambulances) that are required to cover most parts of the whole demand.

Maximum distance (time) between a potential node and a demand node is an important parameter in the EMS location problems. Different values for this parameter have been considered in different countries. The values in urban and rural networks are also different. Another important parameter considered in this context is the required reliability level for the demand served within an acceptable maximum (standard) distance (time).

In this paper, the model presented in the thesis "A model for locating emergency medical service for required reliability levels" [1] is used to determine the location of Emergency Medical Service centres. This model is linear and binary. One of the main variables of this model as many other similar models is "busy fraction", which is calculated as follows: (total estimated time for missions in a demand point) divided by (total number of available ambulances at that point). It should be noted that in this model, the busy fraction is not calculated as an input, but the upper limit is determined for it and the model is designed so that this upper limit is ensured as restrictions of the model for all ambulances. Therefore, if in the desired level of reliability $\alpha$, the minimum number of ambulances $f$, which can cover the demand nodes within the standard distance, is considered, then the reliability of each demand node will be greater than or equal to the pre-specified upper level. The objective function and the constraints of the model are defined as follows:

Objective function: minimize the number of ambulances that should be deployed to provide coverage within the standard time with reliability $\alpha$ for all demand nodes.

Constraints: 1) ensure that the busy fraction of all selected sites (ambulances) will be less than the pre-specified upper bound of ambulance unavailability probability (busy fraction), $\rho .2$ ) Ensure that if a station is sited, at least one ambulance will be positioned in that station. 3) Ensure that a minimum number of ambulances will cover any demand node. 4) Force the binary constraints on the decision variables.

The rest of this paper is organized as follows. Section 2 reviews the revealed literature on reliability-based EMS location problems and the EMS location problems solved by metaheuristic algorithms. Section 3 introduces the Imperialist Competitive Algorithm (ICA). Section 4 describes how the EMS location problem is solved through the ICA. Section 5 presents computational results for two benchmark problems. Section 6 concludes the paper.

\section{RELATED WORKS}

\subsection{Reliability-based EMS location models}

One of the first mathematical programming models that incorporates the ambulance busy fraction is the maximum expected covering location problem (MEXCLP), which has been presented by Daskin [2]; this model assumes an equal busy fraction for all vehicles, which is calculated as an input by dividing the total demand (in hours) by the total servers' capacity (also in hours). Repede and Bernardo [3] developed an extension of the MEXCLP model, called TIMEXCLP, to consider travel time variations throughout a day. Goldberg et al. [4] also 
International Journal of Artificial Intelligence \& Applications (IJAIA), Vol.2, No.4, October 2011

developed a nonlinear model to incorporate the effect of travel time variability into the MEXCLP model by considering the fact that, in reality, each sited vehicle has a specific busy fraction that should be estimated by dividing the duration of the call summoning the vehicle by its available working time. This type of busy fraction has been known in the EMS literature as the 'site-specific busy fraction', as opposed to 'area-specific busy fraction', which is the ratio of the duration of calls throughout a particular area per hour of available service within it. Based on this definition, the busy fraction incorporated in both the MEXCLP and the TIMEXCLP models is an area-specific type. Usually, this type of busy fraction accounts separately for subareas surrounded by the whole study area (e.g. in MALPII and PLSCP developed by Revelle and Hogan [5,6]. Recently, Rajagopalan and Saydam [7] proposed a new approach to locate ambulances that can meet coverage requirements while minimizing expected system-wide response times, inspired by Revelle and Hogan's alpha-reliable p-center model. By assuming each sub-area to be a multi-server loss queue system with Poisson arrivals, Marianov and Revelle [8] proposed QPLSCP to relax the independence assumption in MALPII. In a similar way, various extensions were presented thereafter by integrating the queuing theory and the maximum vehicle availability concept, such as those works presented by Marianov and ReVelle [9] as well as Marianov and Serra [10,11,12]. To provide the reliability requirements for each demand point, Ball and Lin [13] developed a nonlinear constraint that guarantees an upper bound for the unavailability probability of the vehicles serving the demand point. Their model is called Rel-P. Borras and Pastor [14] proposed two adapted visions of the Rel-P model based on the site-specific estimates of busy fractions: a queuing reliability location set covering problem and a binomial reliability location set covering problem.

\subsection{Meta-heuristic algorithms and EMS location problem}

Different meta-heuristic algorithms have been used for solving location models [15-20]. For instance, there are many studies that apply the genetic algorithm (GA) to solve different types of the location problem. Beasley and Chu [21] in 1996 used a genetic algorithm to solve a simple location problem resulting in acceptable results. Aickelin [22] used a special type of genetic algorithm for solving this problem. Jia et al. [23] compared genetic algorithm with other metaheuristic methods.

Simulated annealing (SA) algorithm in solving location model, both alone and in combination with other meth-heuristics have many applications [24-27]. As examples see Murray and Church [28], which uses SA alone, and Marvin et al. [29], which compares the performance of the simulated annealing algorithm with the genetic algorithm and the tabu search algorithm.

A social psychologist, James Kennedy, and an electrical engineer, Russell C. Eberhart, are the owners of the original idea of the Particle Swarm Optimization algorithm. The first simulation of this algorithm was done in 1995 [30-31]. This led them to simulate the behavior of birds to find the seed. Guner and Sevkli [32] used the discrete version of the algorithm to solve location problems. The use of this algorithm showed no significant effect on solving the EMS location problem.

The Imperialist Competitive Algorithm (ICA) has originally introduced by AAtashpaz-Gargari [33], and, up to now, it has been used to deal with various issues. Only over a short period, its application to optimization problems has increased significantly [see 34-38]. The first article about this algorithm has been presented in 2007 [33]. This article presents a brief description of the algorithm and explains how to apply the algorithm as a first attempt for solving the location model problem.

\section{A BRIEF DESCRIPTION OF ICA}

The ICA is a novel global search strategy and inspired by the imperialistic competition based on the human's socio-political evolution [33]. Imperialistic competition forms the core of the 
International Journal of Artificial Intelligence \& Applications (IJAIA), Vol.2, No.4, October 2011 algorithm. This causes all countries to converge to an absolute minimum cost function. Figure 1 shows the flowchart of this algorithm.

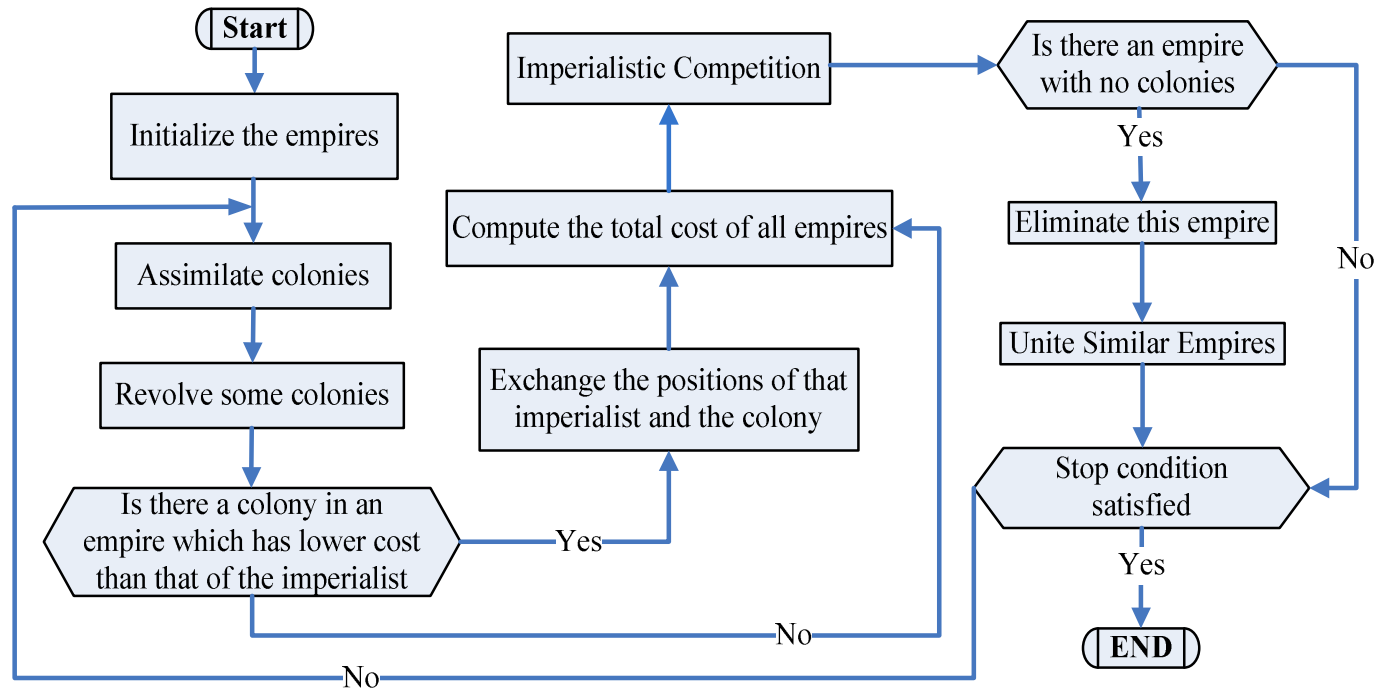

Figure 1. flowchart of ICA [33]

The ICA starts with some countries as initial population, which is classified into two groups. Some of the countries with more power (analogous to the fitness value in the GA) are selected to be the imperialist (analogous to the elite in the GA) and all other countries with less power than the imperialists form colonies of them. The imperialist countries absorb the colonies based on their power using the absorption policy. The total power of an empire depends on both of its constituents, the imperialist country and the colonies. The mathematical expression of this relationship is defined as a power made up of the power of the imperialist country plus a percentage of the average power of the colonies.

After the initial imperialists were formed, the imperialist competition step starts between them. Each imperialist that cannot act successfully in this competition to increase its power (or at least to prevent the loss of its influence) will collapse. Therefore, the survival of an imperialist depends directly on its ability to absorb the rival imperialists' colonies and its ability to rule them out. Consequently, during the imperialist competition, gradually the power of larger imperialist is added and weaker imperialists will be removed. Those empires that want to increase their power will be forced to develop their own colonies. Thus, over time, colonies will be closer to imperialists, and a convergence will be seen. The convergence condition will be achieved when a single imperialist is created along with the colonies whose status are very close to the imperialist country. More details about this algorithm can be found in [20].

\section{APPLICATION OF ICA TO THE EMS LOCATION PROBLEM}

In this section, we seek to apply the ICA in the EMS location problem. Let denote demand nodes by DN and potential stations by PS. The algorithm's steps can be described as the following steps from A to J.

\subsection{Problem inputs}

\section{A. Inputs to the model:}

- Coverage matrix: this is a (PS * DN) matrix with binary variables. If an ambulance $\mathrm{j}$ is within a distance $S$ from demand node $i$, matrix element $(i, j)$ will be one, otherwise it will be zero, 
International Journal of Artificial Intelligence \& Applications (IJAIA), Vol.2, No.4, October 2011

- Demand matrix: this is a $(1 * \mathrm{DN})$ matrix each element of which expresses the amount of demand in a demand node,

- Upper bound of ambulance unavailability probability (busy fraction),

- Minimum number of ambulances which should cover any demand node.

\section{B. Inputs to the ICA:}

- The maximum number of iterations,

- Number of initial countries,

- Number of imperialist countries.

\subsection{Data encoding}

Each country has PS parameter (the search space is equal to PS) that can be zero or one, and as shown in Figure 2 represent parameters of socio-political components of a country. Value of one for a parameter means that the station has been opened (ambulance(s) have been positioned in the station), and zero means that the station has not been opened.

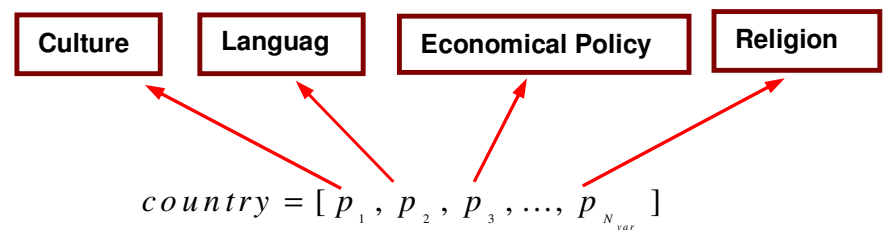

Figure 2. The candidate solutions of the problem, called country, consisting of a combination of some socio-political characteristics such as culture, language and religion

\subsection{Population initialization}

In this step, a parameter is created to represent the number of countries that have been determined through the first iteration. The two following methods have been used for developing initial countries:

- Normal Random Sampling: in this method a random generation function is used such that almost half of the parameters of a country become one and the other half become zero.

- Adjusted Random Sampling: in some cases it is necessary to determine the initial countries so as more parameters have value of one in order to give these parameters the opportunity to converge into their optimal values. This might happen because of the fact that in such cases the need for more non-empty parameters (or positioned ambulances) seems necessary for providing the required service coverage, and predetermination of more ambulances may expedite the algorithm's convergence. Contrarily, in some other cases it might be needed to have more parameters with initial value of zero. In addition, alternatively, answers obtained from the other methods can be used as an input.

\subsection{Fitness evaluation}

In this model the objective function is the sum of characters in each country. In other words, it is equal to the number of parameters that have value of one. To take the constraints into consideration the penalizing strategy has been used, i.e. for violation of each constraint a specific large penalty is added to the objective function. It is to be noted that these penalty 
International Journal of Artificial Intelligence \& Applications (IJAIA), Vol.2, No.4, October 2011

factors should be so greater than the objective function that the algorithm tends to reduce the penalty terms to zero.

In our location model two penalty terms have been adopted. First, a penalty for each uncovered demand point, and second, a penalty for each opened station whose busy fraction exceeds the pre-determined upper bound busy fraction .

\subsection{Initial imperialist countries and their colonies}

According to the fitness function, the cost of each country is calculated, and then all countries are arranged based on the calculated cost. We consider the countries that have lower costs as initial imperialist countries and the rest of the countries as colonies. The colonies are split between the imperialist countries so that the less cost imperialist countries have more chance to attract more colonies.

\subsection{Modeling the assimilation policy}

As mentioned in the previous section, colonial countries are attracted to the imperialist countries. This absorption does not occur directly along the line crosses them, but instead this is done with a small angle, which is an adjustable parameter.

As the original ICA algorithm has been developed for continuous variables during the absorption procedure it also would be possible to produce non-binary values if they are corrected by comparing them with the maximum value of variables (i.e. one) and the minimum value of variables (i.e. zero).

\subsection{Revolution}

In the ICA, colonies are changed in accordance with a revolution rate probability determining if a colony should be kept completely or some of its characteristics should be changed. To this end, usually those countries that are encountered with the revolution phenomenon will be reproduced with a probability of 0.2 (i.e. 0.2 of the number of parameters will possess the value of one).

\subsection{Colonial and imperialist position movement}

Eventually, the costs of the colonies are re-calculated. If there is a colony with a cost less than the cost of its imperialist country, the colony is replaced with the imperialist. This procedure is repeated until all the imperialists are visited.

\subsection{Imperialist competition}

Now, as the next step, we find the weakest imperialist and randomly select one of its colonies to be replaced with it, and then the other imperialist countries will start a competition for the possession of the newly produced colony.

\subsection{Weak imperialist collapse}

After several cycles of weak imperialist countries' takeover, the algorithm will reach at a status where the weak imperialist has no longer a colony. In this case, we will remove the weak imperialist.

\subsection{Stopping criterion}

After a pre-specified number of iterations were repeated, the algorithm stops solving and chooses the optimal solution found so far and display the corresponding scheme.

\section{RESULTS}

To examine the performance of the proposed algorithm, a Matlab code was written, and various parameters were studied. In Section 5.1, two benchmark networks with four demand modes and 
International Journal of Artificial Intelligence \& Applications (IJAIA), Vol.2, No.4, October 2011 two different standard distances were investigated as conventional test networks. The obtained solutions were compared with the exact solution to the problem obtained using GAMS software as well as the results of other meta-heuristic algorithms. (Interested readers can find the details of these algorithms in [1].) To show the capability of the proposed algorithm in large-scale networks and to provide more tangible results, the algorithm was applied to the transportation network of City of Mashhad.

\subsection{Benchmark instances}

In total, eight scenarios (i.e., 2 (coverage distances) $* 4$ (demand modes) $=8$ ) for two networks were considered. The first network has 55 demand nodes and 55 potential nodes created originally by Swain [39] and modified later by Kroll [40]. The second network has 79 demand nodes and 79 potential nodes created originally by Serra [41] and used in many similar works as a test network. In these networks radial distances are considered. In four different modes of demand (in terms of number of calls per day) from the first mode to the fourth mode, the demand increases constructing different problems with different environments for demand patterns and coverage requirements. The higher the figure of scenarios the higher will be the complexity of the problem. The four scenarios were used for each network. To construct coverage matrices two maximum coverage distances (i.e., 1.5 miles and 3 miles) were used. The required reliability level was assumed to be 0.85 in all scenarios. As seen in Table 1 , the proposed algorithm in all scenarios reaches the global solution except for the fourth scenario on 55 node network, and the other algorithms results in the global solution except for the first scenario.

Table 1. Comparisons between the number of required ambulances the proposed algorithm and the other well-known methaheuristic algorithms; GA: genetic algorithms, SA: simulated annealing, and PSO: Particle Swarm Optimization .

\begin{tabular}{|c|c|c|c|c|c|c|c|c|c|}
\hline \multicolumn{2}{|c|}{ Scenario } & 1 & 2 & 3 & 4 & 5 & 6 & 7 & 8 \\
\hline \multirow{4}{*}{\multicolumn{2}{|c|}{ Scenario Description }} & \multicolumn{8}{|c|}{ S (Standard distance, mile) } \\
\hline & & \multicolumn{4}{|c|}{1.5} & \multicolumn{4}{|c|}{3} \\
\hline & & \multicolumn{4}{|c|}{ Demand mode } & \multicolumn{4}{|c|}{ Demand mode } \\
\hline & & 1 & 2 & 3 & 4 & 1 & 2 & 3 & 4 \\
\hline \multirow{5}{*}{55 node network } & Exact Solution & 6 & 10 & 14 & 21 & 2 & 4 & 7 & 12 \\
\hline & GA & 6 & 10 & 14 & 21 & 2 & 4 & 7 & 12 \\
\hline & PSO & 6 & 10 & 14 & 21 & 2 & 4 & 7 & 12 \\
\hline & $\mathrm{SA}$ & 6 & 10 & 14 & 21 & 2 & 4 & 7 & 12 \\
\hline & ICA & 6 & 10 & 14 & 22 & 2 & 4 & 7 & 12 \\
\hline \multirow{5}{*}{79 node network } & Exact Solution & 12 & 12 & 18 & 24 & 5 & 7 & 12 & 18 \\
\hline & GA & 12 & 12 & 18 & 25 & 5 & 7 & 12 & 18 \\
\hline & PSO & 12 & 12 & 18 & 24 & 5 & 7 & 13 & 18 \\
\hline & $\mathrm{SA}$ & 12 & 12 & 18 & 24 & 5 & 7 & 13 & 18 \\
\hline & ICA & 12 & 12 & 18 & 24 & 5 & 7 & 12 & 18 \\
\hline
\end{tabular}

Comparison of convergence trends between the applied algorithms is shown in Figure 3. As shown in this figure, the convergence of the proposed algorithm is considerably faster than those of the other algorithms. This comparison was done for the 79-node network in the second scenario with the fourth demand mode the exact solution of which is 12 . 
International Journal of Artificial Intelligence \& Applications (IJAIA), Vol.2, No.4, October 2011

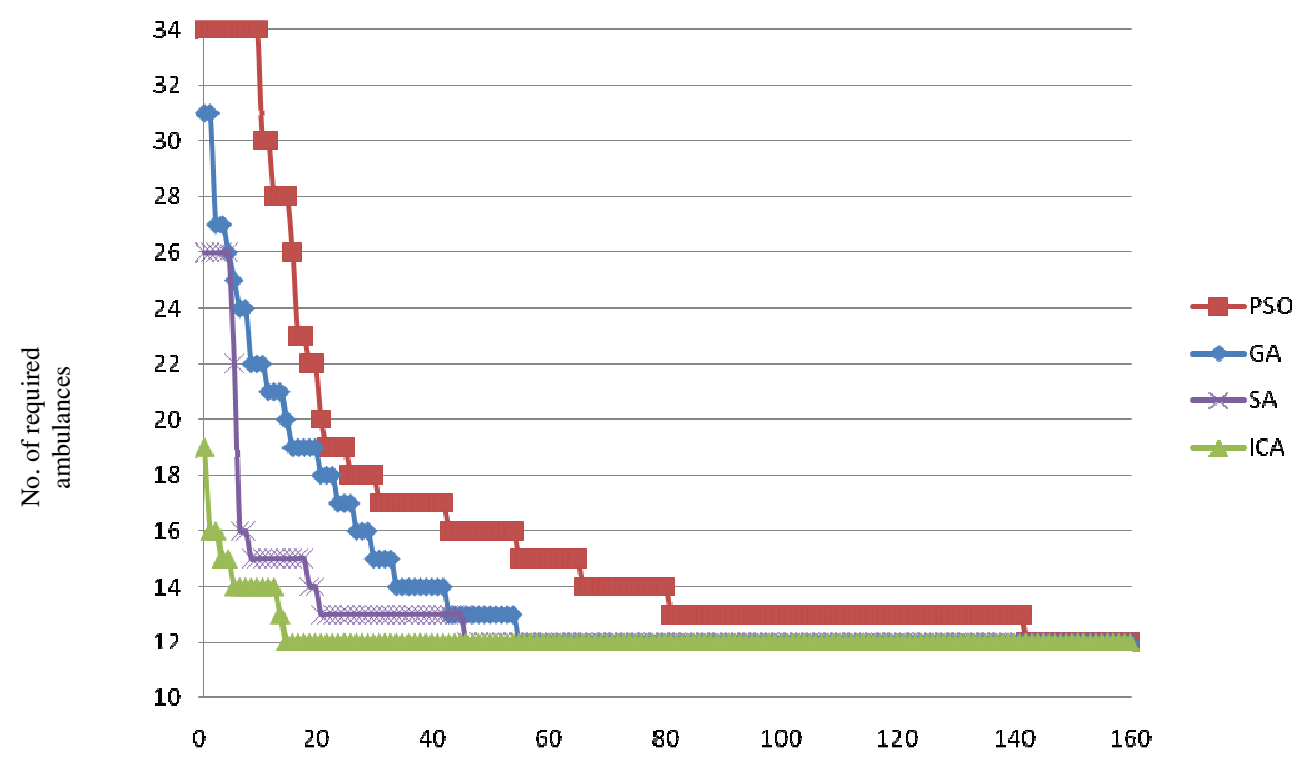

Figure 3. Comparison of cor Iteration Number lgorithm and the other algorithms.

\subsection{Real case-study}

The city of Mashhad is the second largest city in Iran with the population of about 2.5 which sometimes, because of tourism, may exceed 7 million. The basic transpiration network of Mashhad consisting of 253 traffic analysis zones was considered as the potential/demand points. The coverage of demand nodes was determined according to the travel time matrix in a twohour morning peak period. The required reliability level was assumed to be 0.85 as the upper level of reliability. The network is shown in Figure 4.

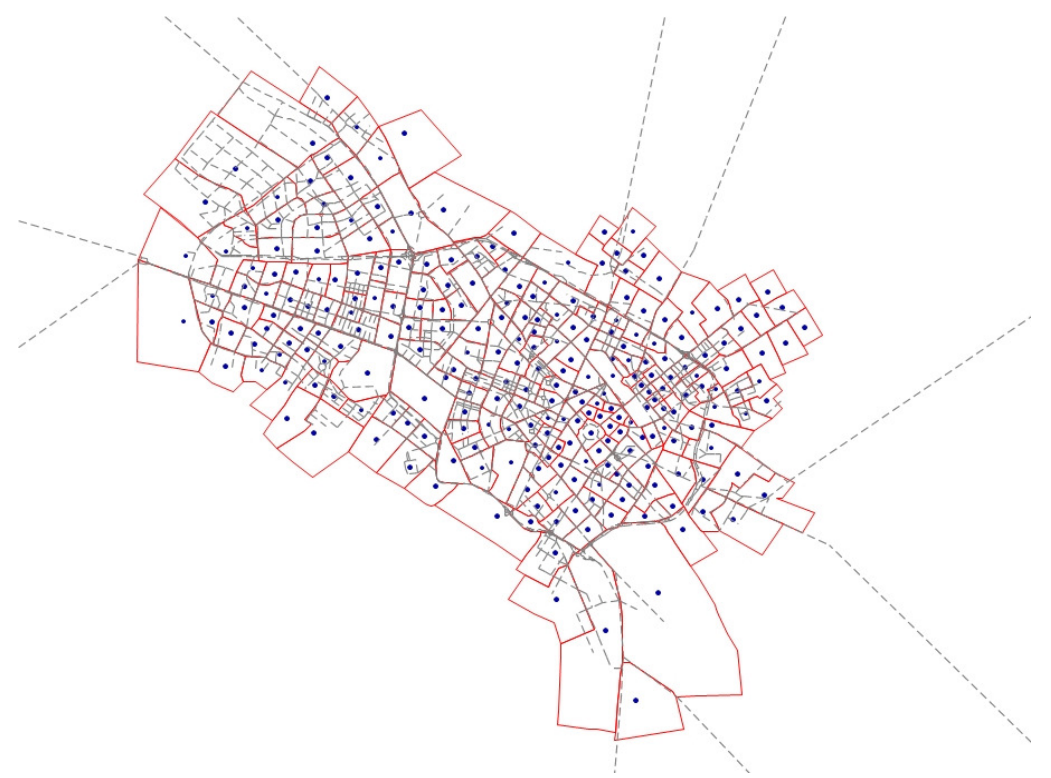

Figure 4. Network and demand nodes of mashhad city 
International Journal of Artificial Intelligence \& Applications (IJAIA), Vol.2, No.4, October 2011 Results of the proposed algorithm and genetic algorithm are shown in Table 2 . This table show that the cpu time needed for the application of the proposed algorithm is about $0.6(=42 / 69)$ of a standard genetic algorithm.

Table 2. Comparisons of the number of required ambulances and the cpu time between ICA and GA.

\begin{tabular}{lll}
\hline & GA & ICA (proposed algorithm) \\
\hline Number of required ambulances & 75 & 75 \\
Run time (second) & 69 & 42 \\
\hline
\end{tabular}

\section{Conclusions}

This paper used the ICA, a recently introduced meta-heuristic algorithm, to solve the EMS location problem. The application of the adapted algorithm to two benchmark networks showed that the solutions obtained from the proposed algorithm compared with those obtained from three well-known meta-heuristic algorithms (i.e., the genetic algorithm, the simulated annealing, and the particle swarm optimization) are competitive and in some cases are better. In addition, the convergence of the ICA is faster than that of the other three meta-heuristic algorithms. Also, the application of the proposed algorithm to the real case study of Mashhad showed that the proposed algorithm is applicable in large-scale networks in the sense of requiring reasonable cpu time as well as reaching promising objective function compared with GA. In recent years, researchers have focused on the ICA for solving continuous problems, but this paper is its first deployment on a discrete problem, i.e. the binary EMS location problem.

\section{REFERENCES}

[1] Moadi, S. (2010) "A model for locating emergency medical service for required reliability levels", Master. thesis, The School of Civil Engineering, Iran University of Science and Technology.

[2] Daskin, M. (1983). "A maximal expected covering location model: Formulation, properties, and heuristic solution", Transportation Science, 17, 48-69.

[3] Repede, J., \& Bernardo, J. (1994). "Developing and validating a decision support system for locating emergency medical vehicles in Louisville, Kentucky", European Journal of Operational Research, 75 (3), 567-581.

[4] Goldberg, J, Dietrich, R., Chen, J., Mitwasi, G., Valenzuela, T., \& Criss, L. (1990b). "Validating and applying a model for locating emergency medical vehicles in Tucson, Arizona" , European Journal of Operational Research, 49 (3), 308-324.

[5] ReVelle, C., \& Hogan, K. (1989a). "The maximum availability location problem" , Transportation Science, 23 (3), 192-200.

[6] ReVelle, C., \& Hogan, K. (1989b). "The maximum reliability location problem and alphareliable p-center problem: Derivatives of the probabilistic location set covering problems", Annals of Operations Research, 18, 155-174.

[7] Rajagopalan, H. K., \& Saydam C. (2009). "A minimum expected response model: Formulation, heuristic solution, and application", Socio-Economic Planning Sciences, 43, 253-262.

[8] Marianov, V., \& ReVelle, C. (1994). "The Queuing probabilistic location set covering problem and some extensions", Socio-Economic Planning Sciences, 167-178.

[9] Marianov, V., \& ReVelle, C. (1996). "The Queueing maximal availability location problem: a model for the siting of emergency vehicles", European Journal of Operational Research, 93 (1), 110-120. 
International Journal of Artificial Intelligence \& Applications (IJAIA), Vol.2, No.4, October 2011

[10] Marianov, V., \& Serra, D. (1998). "Probabilistic, maximal covering location-allocation models for congested systems", Journal of Regional Science, 38 (3), 401-424.

[11] Marianov, V., \& Serra, D. (2001). "Hierarchical location-allocation models for congested systems", European Journal of Operational Research, 135 (1), 195-208.

[12] Marianov, V., \& Serra, D. (2002). "Location-allocation of multiple-server service centers with constrained queues or waiting times", Annals of Operations Research, 111, 35-50.

[13] Ball, M., \& Lin, F. (1993). "A reliability model applied to emergency service vehicle location", Operations Research, 41 (1), 18-36.

[14] Borras, F., \& Pastor, J. T. (2002). "The ex-post evaluation of the minimum local reliability level: An enhanced probabilistic location set covering model", Annals of Operations Research, 111, 51-74.

[15] Correa, ES. Steiner, MTA. Freitas, AA. Carnieri, C. (2004) "Genetic algorithm for solving a capacitated p-median problem", Numer Algorithms, Volume 35, Numbers 2-4, pp. 373-388.

[16] Topcuoglu, H. Corut, F. Ermis, M. Yilmaz, G. (2005) "Solving the un-capacitated hub location using genetic algorithms", Comp \& Oper Res, 32:pp. 467-984.

[17] Kratica, J. Tosic, D. Filipovic, V. Ljubic, I. (2001) "Solving the simple plant location problem by genetic algorithm", Oper Res, 35: pp. 127-142.

[18] Yang, L. Jones, BF. Yang, SH. (2007) "A fuzzy multi-objective programming for optimization of fire station locations through genetic algorithms", Eur J Oper Res, 181: pp. 903-915.

[19] Drezner, Z. (2008) "Extensive experiments with hybrid genetic algorithms for the solution of the quadratic assignment problem", Comp \& Oper Res, 35: pp. 717-736.

[20] Salhi S, GamalMDH. (2003) "A Genetic algorithm based approach for the uncapacitated continuous location-allocation problem", Ann Oper Res 123: pp. 203-222.

[21] Beasley, J.E. \& Chu, P.C. (1996) "A genetic algorithm for the set covering problem", European Journal of Operational Research, 94, pp. 392-404.

[22] Aickelin, U. (2002) "An indirect genetic algorithm for set covering problems", Journal of the Operational Research Society, 53, pp. 1118-1126.

[23] Jia, H. Ordonez, F. Dessouky, M. (2007) " Solution approaches for facility location of medical supplies for large-scale emergencies", Computers \& Industrial Engineering, 52, pp. 257-276.

[24] Antunes, A. Peeters, D. (2000) " A dynamic optimization model for school network planning",Socio Econ Plan Sci, 34(2): pp. 101-120.

[25] Hansen, P. Jaumard, B. Taillard, E. (1998) "Heuristic solution of the multi source Weber problem as a p-median problem",Oper Res Lett, 22: pp. 55-62.

[26] Alves, M. L., \& Almeida, M. T. (2002) "Simulated annealing algorithm for the simple plant location problem: A computational study",Rev. Invest. 12. 3. Aydin, M.E. and T.C. Fogarty.

[27] Mavridou, T. Pardalos, PM. (1997) "Simulated annealing and genetic algorithms for the facility layout problem: A survey",Computat Optimiz Appl, 7: pp. 111-126.

[28] Murray, AT. Church, RL. (1996) "Applying simulated annealing to location-planning models",J Heuristics, 2: pp. 31-53.

[29] Marvin, A. Arostegui, Jr.a. Sukran, N. Kadipasaoglub, Basheer, M. Khumawala. (2006) "An empirical comparison of Tabu Search, Simulated Annealing, and Genetic Algorithms for facilities location problems",Int. J. Production Economics, 103: pp. 742-754.

[30] Kennedy, J.and Eberhard, R. C. (1995) "Particle Swarm Optimization,Proc. of IEEE Int'l Conf. on Neural Networks, Piscataway, , NJ, USA, pp. 1942-1948.

[31] Eberhart, R. Kennedy, C. (1995) "A new optimizer using particle swarm theory", in Proc. 6th Int. Symp. Micro Machine and Human Science (MHS), pp. 39-43.

[32] Guner, A. and Sevkli, M. (2008) "A Discrete Particle Swarm Optimization Algorithm for Uncapacitated Facility Location Problem", Journal of Artificial Evolution and Applications, Vol. 2008, No. 861512, , pp. 1-9.

[33] Atashpaz-Gargari, E. and C. Lucas. (2007) "Imperialist Competitive Algorithm: An Algorithm for Optimization Inspired by Imperialistic Competition" , IEEE Congress on Evolutionary Computation (CEC 2007). pp 4661-4667. 
International Journal of Artificial Intelligence \& Applications (IJAIA), Vol.2, No.4, October 2011

[34] Caro Lucas, Zahra Nasiri-Gheidari, Farid Tootoonchian. (2010) "Application of an imperialist competitive algorithm to the design of a linear induction motor", Energy Conversion and Management, Elsevier, pp 1407-1411.

[35] Tushar Jain, M.J. Nigam. (2010) "Synergy of evolutionary algorithm and socio-political process for global optimization", Expert Systems with Applications 37 , pp. 3706-3713.

[36] Shokrollahpour E. Zandieh M. Behrouz Dorri B. (2010) " A novel imperialist competitive algorithm for bi-criteria scheduling of the assembly flowshop problem", International Journal of Production Research, 103: pp. 742-754.

[37] Ramin Rajabioun, Esmaeil Atashpaz-Gargari, Caro Lucas. (2008) " Colonial Competitive Algorithm as a Tool for Nash Equilibrium Point Achievement", Springer LNCS, Lecture Notes in Computer Science, Volume 5073/2008, pp. 680 - 695.

[38] Atashpaz-Gargari, E. Hashemzadeh, F. Rajabioun, R. and Lucas, C. (2008) "Colonial Competitive Algorithm, a novel approach for PID controller design in MIMO distillation column process", International Journal of Intelligent Computing and Cybernetics, Vol. 1 No. 3, pp. 337355, pp.

[39] Swain R. (1971)"A descomposition algorithm for a class of facility location problems", Ph.D. thesis, Cornell ,University, Ithaca, NY.

[40] Kroll P. (1988) "The multiple-depot, multiple-tour and multiple-stop delivery problem", Ph.D. dissertation, The Johns Hopkins University, Baltimore, MD.

[41] Serra D. (1989) "The pq-median problem: Location and districting of hierarchical facilities" , Ph.D. thesis, Department of Geography and Environmental Engineering, John Hopkins University. 